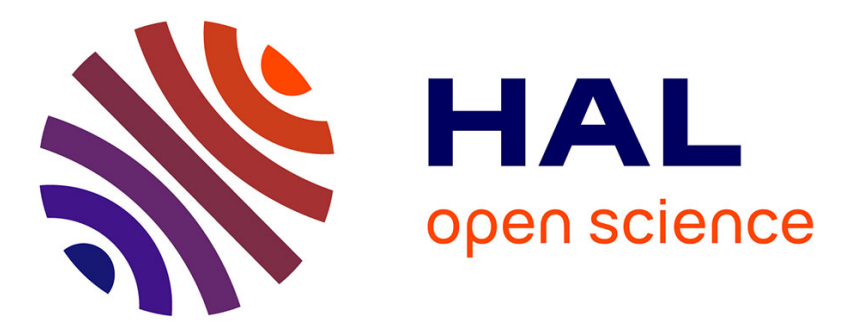

\title{
Toward RCS Magnitude Level Coding for Chipless RFID
}

Olivier Rance, Romain Siragusa, Pierre Lemaitre-Auger, Etienne Perret

\section{To cite this version:}

Olivier Rance, Romain Siragusa, Pierre Lemaitre-Auger, Etienne Perret. Toward RCS Magnitude Level Coding for Chipless RFID. IEEE Transactions on Microwave Theory and Techniques, 2016, 64 (7), pp.2315-2325. 10.1109/TMTT.2016.2562625 . hal-02066610

\section{HAL Id: hal-02066610 https://hal.science/hal-02066610}

Submitted on 12 May 2020

HAL is a multi-disciplinary open access archive for the deposit and dissemination of scientific research documents, whether they are published or not. The documents may come from teaching and research institutions in France or abroad, or from public or private research centers.
L'archive ouverte pluridisciplinaire HAL, est destinée au dépôt et à la diffusion de documents scientifiques de niveau recherche, publiés ou non, émanant des établissements d'enseignement et de recherche français ou étrangers, des laboratoires publics ou privés. 


\title{
Toward RCS Magnitude Level Coding for Chipless RFID
}

\author{
O. Rance, R. Siragusa, P. Lemaître-Auger, and E. Perret, Senior Member IEEE
}

\begin{abstract}
Hybrid coding techniques have been proposed recently to improve the coding capacity of Chipless Radiofrequency identification (RFID) tag. The article examines the possibility to code information thanks to the magnitude level of the radar cross section (RCS) in addition to the more classical technique of frequency position. Single-layer tags based on Cfolded dipoles are designed to have different magnitude levels. A magnitude span of up to $15.2 \mathrm{~dB}$ is obtained for coupled resonators. A magnitude resolution of $3.5 \mathrm{~dB}$ is evaluated for practical applications based on the measurement of the realized tags in different configurations. The problem of tags applied to an unknown object is considered and a compensation technique is proposed for an object similar to a thin dielectric plate.
\end{abstract}

Index Terms - chipless RFID, RCS, scatterer, magnitude level

\section{INTRODUCTION}

$\mathrm{C}$ Chipless radio frequency identification (RFID) is an emerging identification technology compatible with printing techniques that can considerably decrease the price compared to current RFID systems that employ tags with silicon chips[1]-[3]. This passive technology can potentially compete with the optical barcode [4], offering new capabilities such as increased read range and reading of coated tags $[5],[1]$.

An important problem still present in the design of a chipless tag is to increase the coding capacity of the tag even as maintaining a size of a credit card. For that purpose, several techniques are proposed in literature. Today, chipless RFID tags may thus be classified according to the coding technique they use [4]. They are divided into two main categories: time coding and frequency coding. Time domain based tags [6]-[8] have simple calibration procedure and reasonable readable ranges up to $80 \mathrm{~cm}$, but the bit encoding capacity is low [9]. The best coding density is generally obtained with frequency position (FP) coding [2], [10]-[12] which is based on the control of resonances at certain frequencies of the spectrum. Each resonance is associated to a physical scatterer and the coding capacity is then related to the number of scatterers present on the tag and to the permitted frequency band.

O. Rance, R. Siragusa, P. Lemaître-Auger, E. Perret are with the Laboratoire de Conception et d'Intégration des Systèmes (LCIS) - Grenoble Institute of Technology, Valence, France (e-mail: olivier.rance@lcis.grenoble-inp.fr; pierre.lemaitre-auger@lcis.grenoble-inp.fr romain.siragusa@lcis.grenobleinp.fr; etienne.perret@lcis.grenoble-inp.fr). E. Perret is also with the Institut Universitaire de France, Paris, France.
TABLE I

COMPARISON OF ENCODING TECHNIQUES FOR CHIPLESS RFID

\begin{tabular}{|c|c|c|c|c|c|c|}
\hline \multicolumn{2}{|c|}{$\begin{array}{l}\text { Coding } \\
\text { Technique }\end{array}$} & \multicolumn{2}{|c|}{ Resonator Type } & \multirow{2}{*}{ 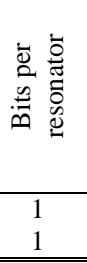 } & \multirow{2}{*}{ 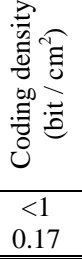 } & \multirow{2}{*}{ 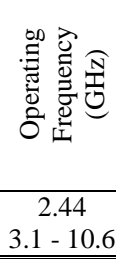 } \\
\hline & Time Domain & $\begin{array}{l}{[7]} \\
{[8]}\end{array}$ & $\begin{array}{l}\text { SAW } \\
\text { Delay line }\end{array}$ & & & \\
\hline & $\begin{array}{l}\text { Frequency } \\
\text { Domain }\end{array}$ & $\begin{array}{l}11] \\
{[10]} \\
{[11]} \\
11]\end{array}$ & $\begin{array}{l}\text { Spiral } \\
\text { Slot } \\
\text { C-shape } \\
\end{array}$ & $\begin{array}{l}1 \\
1 \\
1 \\
\end{array}$ & $\begin{array}{l}0.61 \\
4.17 \\
1.14 \\
\end{array}$ & $\begin{array}{c}3.1-7 \\
3.1-10.6 \\
2-4 \\
\end{array}$ \\
\hline & $\begin{array}{l}\text { Frequency } \\
\text { Dual Band }\end{array}$ & $\begin{array}{l}13] \\
{[14]}\end{array}$ & $\begin{array}{l}\text { Stub loaded } \\
\text { Stepped } \\
\text { impedance }\end{array}$ & $\begin{array}{c}1.5 \\
2\end{array}$ & $\begin{array}{l}<1 \\
7.9\end{array}$ & $\begin{array}{l}2.5-5.5 \\
3.1-10.6\end{array}$ \\
\hline & $\begin{array}{c}\text { Frequency } \\
\text { Dual polarized } \\
\end{array}$ & [15] & Slot & 1 & 5.88 & $3.2-9.6$ \\
\hline \multirow{4}{*}{ 营 } & $\begin{array}{l}\text { Frequency - } \\
\text { phase }\end{array}$ & [17] & C-shape & 4.6 & 2.86 & $2.5-7.5$ \\
\hline & $\begin{array}{l}\text { Frequency - } \\
\text { angle }\end{array}$ & [20] & $\begin{array}{l}\text { Stepped } \\
\text { impedance }\end{array}$ & 3 & 0.52 & $1.8-2.2$ \\
\hline & $\begin{array}{l}\text { Frequency - } \\
\text { bandwidth }\end{array}$ & [21] & $\begin{array}{l}\text { Dipole, } \\
\text { rect. ring, } \\
\text { rect. patch }\end{array}$ & 4 & 3.6 & $2-5$ \\
\hline & $\begin{array}{l}\text { Frequency- } \\
\text { magnitude }\end{array}$ & 苛 & C-shape & 3 & 1.25 & $2-5$ \\
\hline
\end{tabular}

A significant increase can be achieved if each resonator codes several different states. This simple idea has been exploited in different ways to enhance coding capacity. Recent work are using both the fundamental and first harmonic for encoding [13], [14]. A second method is to use dual polarized tags, with a different code for each polarization, allowing spectral efficient coding [15],[16]. Another approach called hybrid coding is to use several physical quantities to encode information. Each quantity must be controlled by independent geometric parameters for a maximum benefit of the approach. Several types of hybrid coding tags have been reported in literature: FP - phase [17], FP - group delay [18], [19], FP angle [20], FP - bandwidth [21], FP - magnitude [22], [23]. These different coding techniques are compared in terms of bit per single resonator, coding density and spectral efficiency in Table I.

The magnitude hybrid coding principle has been introduced in [22] to enhance coding density of resonant scatterers having low quality factors which limits the FP coding efficiency. This happens for tags printed on high loss substrate like paper. A resistive strip with variable width is included in the resonator to control the level of the response. The magnitude range obtained in this manner is relatively low 


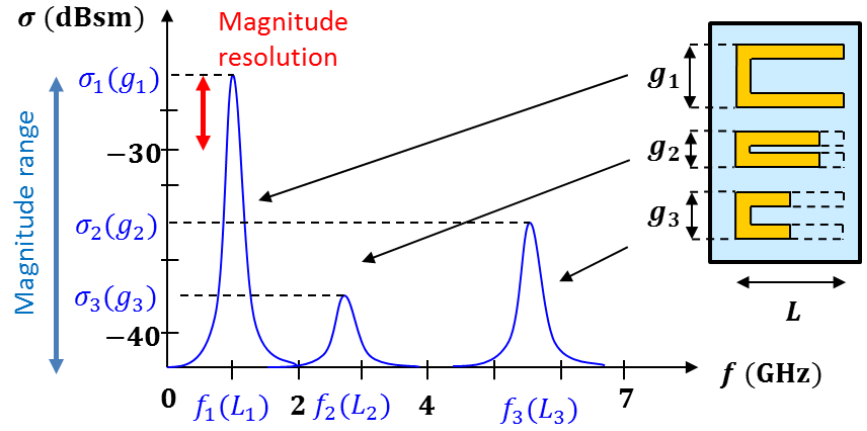

Fig. 1. RCS magnitude level coding principle. The tag is composed of resonant scatterers, which results in peaks in its spectrum. The objective is to identify the RCS magnitude level of each scatterer. The magnitude range and resolution $(3 \mathrm{~dB})$ and may not be sufficient for practical applications. Another implementation of the magnitude coding has been reported in [23] offering larger magnitude range $(12 \mathrm{~dB})$. The magnitude is controlled thanks to the polarization mismatch between the tag and the antennas. The tags include a ground plane and are therefore not impacted by the object on which they are applied on. However, they are not compatible with a printing realization process.

In this article, we examine the feasibility of the hybrid coding technique where the scatterers code different radar cross section (RCS) magnitude levels in addition to the more traditional FP technique (Fig. 1). A comprehensive study is presented that goes from the theoretical aspect to the practical implementation. The scatterer used for the design of the present article is a C-folded dipole (Fig. 1) [11], [17]. It is a single-layer structure, which is potentially compatible with printing process. Two geometric parameters $L$ and $g$ control respectively the resonance frequency and the magnitude of the response. Because the magnitude of the response is limited to a small range $(2.7 \mathrm{~dB})$ with only one resonator, to obtain a larger range $(15.2 \mathrm{~dB})$, the resonators have to be coupled. The result of such a coupling is that the magnitude level at one resonant frequency cannot be addressed individually for each resonator. This allows a limited gain of 2 bits per tag. We consider, though, that the tags realized in this manner are sufficient to study the applicability of the general method. Indeed, no real examination of practical applicability of the method has been carried out in previous works [22], [23].

As a matter of fact, magnitude coding presents difficulties in practice because the magnitude of a resonator is particularly sensitive to noise and can be influenced by various factors. For example, the object on which the tag is applied to, has its own RCS, which can skew the results. Other factors such as the distance between the tag and the reader, the attenuation caused by the propagation medium (obstacle between the tag and the reader) have to be considered for practical implementation. The purpose of this article is to assess the feasibility of the magnitude coding approach and to determine a sufficient magnitude resolution for practical measurements. The problem of the additional contribution of the object is also considered and a compensation technique is proposed for object similar to thin dielectric plates.

The article is organized as follows. In section II, the design of the tag is presented. Particular attention is paid to the effect of couplings on the magnitude level. In section III, different factors that can modify the RCS magnitude of the tag are investigated. The magnitude resolution necessary to achieve a robust design is evaluated based on measurement results. In section IV, a compensation technique for a tag applied on an unknown dielectric plate of small width is proposed and validated by measurement results.

\section{DESIGN OF THE TAG}

The "RF encoding particles" (REP) approach is used [1] and the elementary particle chosen for coding is the C-folded dipole (Fig. 2-a), which has been already studied since a few years [11], [17]. The first step of the design is to establish the relations between the geometrical parameters of a single scatterer and the corresponding electromagnetic (EM) signature (Fig. 2). For that purpose, two complementary models of the scatterer are proposed. The model has to account for radiation properties of the scatterers to determine the magnitude level, which is not classically done for chipless RFID. To achieve an important coding capacity, it is necessary to integrate several scatterers within the same tag [9] which can generate important couplings. We will show in particular how couplings can enhance the RCS magnitude level of the scatterers and eventually increase the possible magnitude span of the entire tag.

\section{A. Single Resonator}

In [17], the C-folded dipole has been identified among other single layer resonators to have a good tradeoff in terms of quality coefficient $(\mathrm{Q}=65)$, RCS level $(-30 \mathrm{~dB})$, and frequency range $(2.5 \mathrm{GHz}$ to $7.5 \mathrm{GHz})$ with a reduced size $(L \simeq \lambda / 4)$.

A model of the resonator was first introduced in [17]. The C-folded dipole can be seen as a transmission line (TL) with both a shortened (SC) and an open termination (OC) (Fig. 2b). It thus behaves like a quarter wavelength resonator. The fringing fields at the end of the TL can be taken into account by introducing a supplementary length $\Delta l$, which is a function of the transverse geometry. For a given gap $g, \Delta l$ remains constant and can be readily evaluated by simulation fitting. The resonance frequency is given by:

$$
f_{r}=\frac{c}{4(L+\Delta L) \sqrt{\varepsilon_{e}}},
$$

where the effective relative permittivity $\varepsilon_{e}$ is the one of a

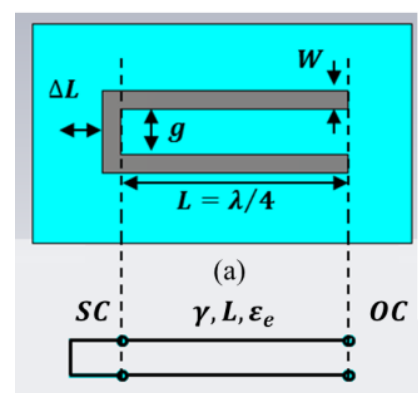

(b)

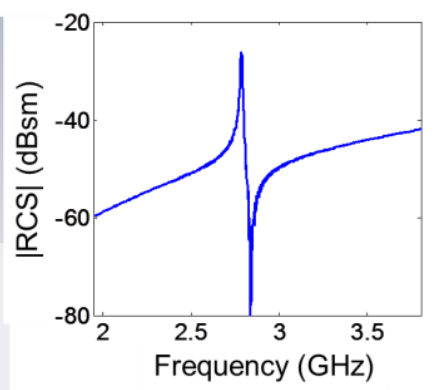

(c)
Fig. 2. (a) Pattern of the C-folded dipole with (b) its equivalent TL circuit where SC stands for short circuit and $\mathrm{OC}$ for open circuit and (c) corresponding EM signature. It behaves like a quarter wavelength resonator. 
coplanar stripline [24] and then depends on the gap value $g$, the width $W$ and thickness $t$ of the strips, as well as the substrate thickness $h$ and permittivity $\varepsilon$. This model enables to set the resonant frequency of a single scatterer by adjusting $L$. However, it does not provide information about the far-field radiation characteristics which is of interest for magnitude coding.

A complementary model is adapted from [25] to relate the RCS magnitude to the value of the gap $g$. If $g$ is small compared to the guided wavelength $\lambda$ and fringing fields are neglected, the structure can be assimilated to a cavity of same geometry but with a magnetic wall at the open termination. The principal resonant mode of this cavity is obtained when $L=\lambda / 4$, which overlaps the TL result. The field stored between the two strips for $-\lambda / 8 \leq z \leq \lambda / 8$ is given by (2):

$$
\overrightarrow{\boldsymbol{E}_{\boldsymbol{s}}}=j I \eta_{0} \sin [2 \pi(z / \lambda+1 / 8)] \widehat{\boldsymbol{x}}
$$

where $\eta_{0}$ is the free space impedance and $I$ is the total current through the short arm. The coordinate system consistent with [25] is indicated in Fig. 3-a. The E-field distribution has been obtained by full wave simulation (CST microwave studio) and is compared to the model prediction in Fig. 3 with good agreement. A drop in the stored field value is observed in Fig. 3-b at the open termination $(z>0.12 \lambda)$ in simulation compared to theory, which is due to fringing fields.

For small values of $g$, the electrical current density over the conductors for the principal mode is given by (3):

$$
\overrightarrow{\boldsymbol{J}}(r)=\left\{\begin{array}{c} 
\pm I \cos [2 \pi(z / \lambda+1 / 8)] \hat{\boldsymbol{z}} \\
I \hat{\boldsymbol{x}}
\end{array}\right.
$$

The constant current distribution in the short arm $(I \widehat{\boldsymbol{x}})$ is due to the small electrical length. Thus its behavior is similar to an infinitesimal dipole. The opposite current flow in the long strips indicates a small cross-polarization value. The polarization of antennas has then to be aligned with the direction of the short arm $(\widehat{\boldsymbol{x}})$ to ensure optimal response of the resonator. The current density along the interior path obtained by full wave simulation is compared to the model prediction in

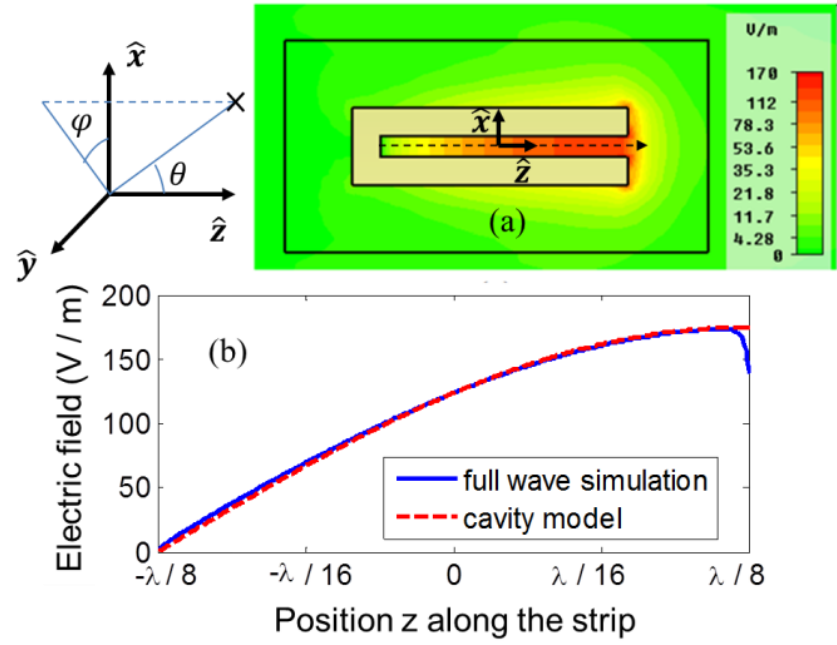

Fig. 3. (a) E-field stored between the strips at resonance for a C-folded dipole applied on a dielectric substrate obtained by full wave simulation (CST Microwave Studio). (b)

Comparison between the full wave simulation and the cavity
Fig. 4) with good agreement. The current densities on the strips (the absolute values are represented in Fig. 4-a,b) are opposite and are following a cosine distribution for $z>$ $-0.1 \lambda$. A sudden increase of the current density $(3.9 \mathrm{~A} / \mathrm{m}$ to $7.8 \mathrm{~A} / \mathrm{m}$ for the bottom strip) can be seen for $z<-0.1 \lambda$. This is related to wedge effects and to the thickness of the strip (the calculation path is taken at the middle of the strip width but different density values are observed if we are closer to the substrate). We can also note that the current density on the small arm (Fig. 4-c) is not exactly constant and shows maxima at the wedges. A relative variation of $11 \%$ is observed compared to the mean value.

Using the current distribution of (3), the far-field can be calculated with respect to the direction of observation [25] and is given by (4):

$$
\left\|\overrightarrow{\boldsymbol{E}_{\boldsymbol{f f}}}\right\|^{2}=\frac{\eta_{0}{ }^{2}(g I)^{2}}{4 \lambda^{2} r^{2}} \operatorname{sinc}^{2}\left(\alpha_{1}\right) \operatorname{sinc}^{2}\left(\alpha_{2}\right),
$$

where:

$$
\begin{aligned}
& \alpha_{1} \equiv \pi(g / \lambda) \sin \theta \sin \varphi, \\
& \alpha_{2} \equiv \pi(g / \lambda) \sin \theta \cos \varphi,
\end{aligned}
$$

where $r$ is the distance to the observation point and $\theta$ and $\varphi$ are angles of observation indicated on Fig. 3. It is readily seen that the field intensity depends on the length $g$. As $g$ is small compared to wavelength the scatterer can be considered isotropic [25]. The re-radiation pattern of such scatterer has been obtained by full wave simulation in [3] and is in good agreement with the theoretical prediction.

The RCS magnitude level at the resonant frequency $3.5 \mathrm{GHz}$ has been extracted from full wave simulations and is compared to the one obtained from the cavity model in Fig. 5. We observe a marked discrepancy concerning the exponent value of $g$ between simulation (black line) and theory (red line). This can be explained by the differences between the idealized structure proposed in [25] and the current scatterer:

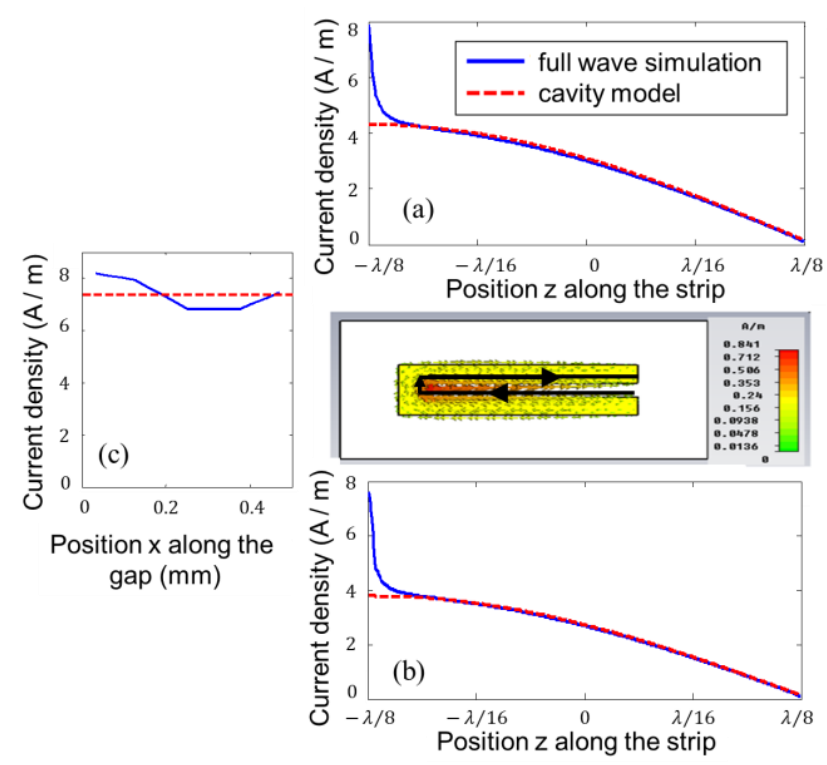

Fig. 4. Current density along the interior path at resonance. Comparison between full wave simulation (CST Microwave Studio) and cavity model. 
presence of the substrate, strip width comparable to the gap value, and thickness of the strips much lower than the gap value. Nevertheless, a similar qualitative behavior is observed: the RCS is sensitive mainly to the value of $g$ with respect to the wavelength. By analogy to (4), a variation model of the form $\sigma=a . g^{b}$ is assumed. The parameters $a$ and $b$ are adjusted to best fit the full wave simulation at a least square sense. When $g$ belongs to the infinitesimal dipole region $(g<\lambda / 50$, i.e. $g<L / 12.5)$ the obtained values are $a=$ $0.11 \mathrm{~m}^{2}$ and $b=0.5$ (dashed blue line). When $g$ belongs to the small dipole region $(g<\lambda / 10$, i.e., $g<L / 2.5)$, the obtained values are $a=0.0075 \mathrm{~m}^{2}$ and $b=0.16$ (dashed green line). For a different value of $L$, a similar diagram can be easily drawn from only three simulations $(g<$ $L / 12.5, g=L / 12.5$, and $g>L / 12.5$ ), which can then be used for design.

The quality factor $Q_{s p}$ (power radiated into space) of the theoretical scatterer has been studied in [25] for $g$ small compared to wavelength and is given by (6):

$$
\frac{1}{Q_{s p}} \simeq\left(\frac{g}{\lambda}\right)^{2} \text {. }
$$

In presence of a substrate, and for lossy conductor, the total quality factor is modified [3]:

$$
\frac{1}{Q}=\frac{1}{Q_{s p}}+\frac{1}{Q_{d}}+\frac{1}{Q_{c}}
$$

where $Q_{d}$ and $Q_{c}$ denote dielectric and conductor quality factors corresponding to the power dissipated by dielectric loss, and dissipated by conductor loss, respectively.

For the latter a variation range of $g$ of $0.5 \mathrm{~mm}$ to $5 \mathrm{~mm}$ is considered, which is compatible with a printing realization process. The corresponding magnitude range is $2.75 \mathrm{~dB}$ for a single resonator, which is unfortunately not sufficient to perform a magnitude coding. In the following section, we will seek for a larger range by examining the effect of couplings.

\section{B. Couplings}

To increase the coding capacity, several scatterers are integrated together within the tag. For the present article, five $\mathrm{C}$-folded dipoles are disposed in a row in a tag of dimension

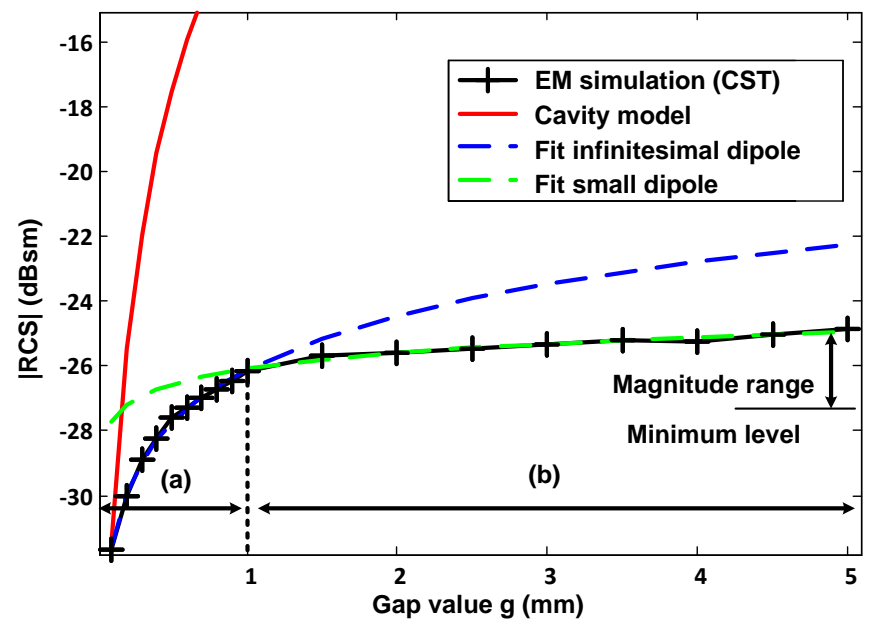

Fig. 5. RCS magnitude level at the resonant frequency $3.5 \mathrm{GHz}$ with respect to the gap value $g$. (a) infinitesimal dipole region, (b) small dipole region. The length and the width of the strip are constant: $L=12.7 \mathrm{~mm}$, and $W=1 \mathrm{~mm}$.
$30 \mathrm{~mm} \times 50 \mathrm{~mm}$ (Fig. 6). It generates strong couplings and the overall response of the tag can sensibly vary from the single resonator configuration.

In [9], the authors compared the effect of couplings with respect to the configuration to perform frequency coding. They compared the RCS of five resonators of same gap value $g$ and spacing $p$, disposed in columns (vertical arrangement) (Fig. 6) or in row (horizontal arrangement). A slight frequency offset of maximum $1.5 \%(30 \mathrm{MHz})$ compared to the single resonator characteristic was observed. This was seen as a supplementary length $\Delta L_{c}$ due to couplings and it was compensated by the length of the resonators. For a different code, that is, small variations of the $L_{i}$ values, the couplings remain similar and holding a constant value of $\Delta L_{c}$ is enough to have negligible frequency deviation.

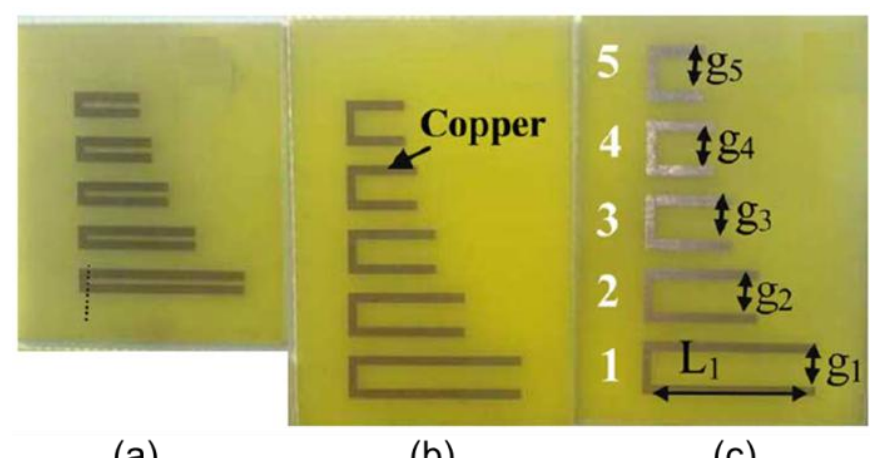

(a)

(b)

(c)

Fig. 6. Realized tags: (a) tag 1, (b) tag 3, (c) tag 4. Each tag is composed of five C-folded dipole of same gap value within a maximum surface of $30 \mathrm{~mm}$ $\times 50 \mathrm{~mm}$. The resonant frequencies of the scatterers are $2.5 \mathrm{GHz}, 3.5 \mathrm{GHz}$, $4.5 \mathrm{GHz}$, and $5.5 \mathrm{GHz}$.

TABLE II

\begin{tabular}{|l||cccccc|}
\multicolumn{7}{c|}{ PARAMETER VALUES OF THE REALIZED TAGS } \\
\hline $\mathrm{mm}$ & $g$ & $L_{1}$ & $L_{2}$ & $L_{3}$ & $L_{4}$ & $L_{5}$ \\
\hline \hline Tag 1 & 0.5 & 18.4 & 12.7 & 9.7 & 7.8 & 6.4 \\
Tag 2 & 1.5 & 18.9 & 12.7 & 9.4 & 7.4 & 6.1 \\
Tag 3 & 2.5 & 19.1 & 12.5 & 9.2 & 7.1 & 5.7 \\
Tag 4 & 3.5 & 19.1 & 12.1 & 8.9 & 6.7 & 5.4 \\
$f_{r} G H z$ & & 2.5 & 3.5 & 4.5 & 5.5 & 6.5 \\
\hline
\end{tabular}

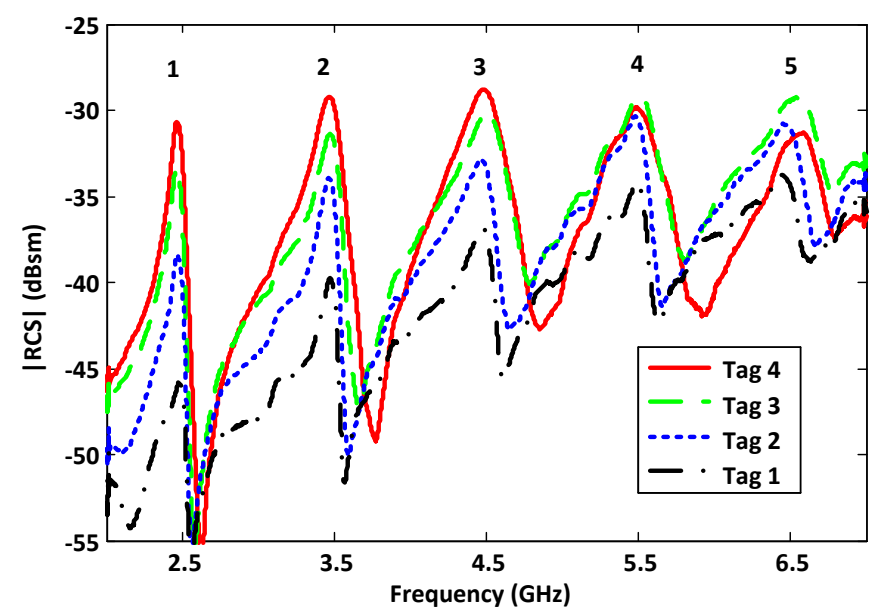

Fig. 7. EM signature measurements of the realized tags. Each tag spectrum is composed of 5 resonant peaks which correspond physically to the $5 \mathrm{C}$ folded dipoles. The tags are designed to have the same resonant frequencies. Each tag shows a different RCS magnitude level which is set by adjusting the value of the gap $g$. Values of the geometrical parameters are given in Table II. Only the 3 first peaks are showing a sufficient magnitude span for 
A parametric study was performed to determine the magnitude sensitivity of the peaks to the gap value in presence of couplings. Similar to [9], both horizontal and vertical configurations were tried. For the horizontal configuration, an important variation was observed when the value of the gap of all resonators was kept identical. Four tags showing different magnitude values have been realized and measured (Fig. 7, measurement setup is detailed later on the in the article) to illustrate this phenomenon. Parameter values are given in Table II. The tags are realized on a FR4 substrate of permittivity $\varepsilon=4.6$ and thickness $h=0.8 \mathrm{~mm}$. The tags are designed to have the same resonant frequencies at $2.5 \mathrm{GHz}$, $3.5 \mathrm{GHz}, 4.5 \mathrm{GHz}, 5.5 \mathrm{GHz}$, and $6.5 \mathrm{GHz}$. The variation of the gaps induces a frequency shift, which is compensated by adjusting the value of $L$ (see Table II). Doing this, a frequency shift less than $50 \mathrm{MHz}$ is obtained from one tag to another. The spacing between the scatterers is held constant $(p=$ $2 \mathrm{~mm}$ ). The first three peaks show the most significant span (respectively $15.2 \mathrm{~dB}, 10.6 \mathrm{~dB}$, and $8.1 \mathrm{~dB}$ ) and could be used for coding in magnitude.

A second study is done to evaluate the response when the gap of only one resonator is varied, even as all other parameters are kept constant. The gap value $g_{2}$ (Fig. 7) of the scatterer number 2 is varied from $0.5 \mathrm{~mm}$ to $3.5 \mathrm{~mm}$. The other geometrical parameters are held constant and correspond to tag2 (see Table II). The corresponding magnitude of the peaks is reported in Fig. 8. In this case, the variation is less pronounced than expected. The magnitude span of the second peak (varying one) is $\Delta \sigma_{2}=1.7 \mathrm{~dB}$. It is not larger than for the single scatterer configuration $(2.4 \mathrm{~dB})$. An interesting fact is that the surrounding scatterers show sensitivity that is comparable to the varying one (peak 1: $0.6 \mathrm{~dB}$, peak 3: $1.8 \mathrm{~dB}$, peak 4: $-2.1 \mathrm{~dB}$ ). It shows that the resonators are strongly coupled.

It implies two major difficulties for the design. Firstly, there is no accurate model to describe the effect of couplings for such structures and the design would then be based on empirical techniques. For example, an attempt to model couplings for similar scatterers was done in [26] but with limited success. Secondly, in practice, it implicates that the RCS magnitude levels of the peaks cannot be set independently from one to another. In other words, for a given tag, all peaks will have a comparable magnitude level and for a large resolution, all the peaks will code the same magnitude information. This constitutes a clear limitation in the additional coding capacity allowed by magnitude control for this structure.

\section{Coding capacity}

The design is limited to the case of identical gap values (Fig. 6). If we consider that we can have four different magnitude levels (see Fig. 7), an increase of 2 bit is obtained. For the initial tag [17], considering only the FP coding, 6 different frequency positions are considered for each resonator leading to a total coding capacity of $\log _{2}\left(6^{5}\right)=13$ bits. Due to the coupling between the resonators, only a gain of 2 bit is obtained with the magnitude coding which leads to a total coding capacity of 15 bits within a tag of size $3 \mathrm{~cm} \times 4 \mathrm{~cm}$. One can easily imagine that other designs, based on other

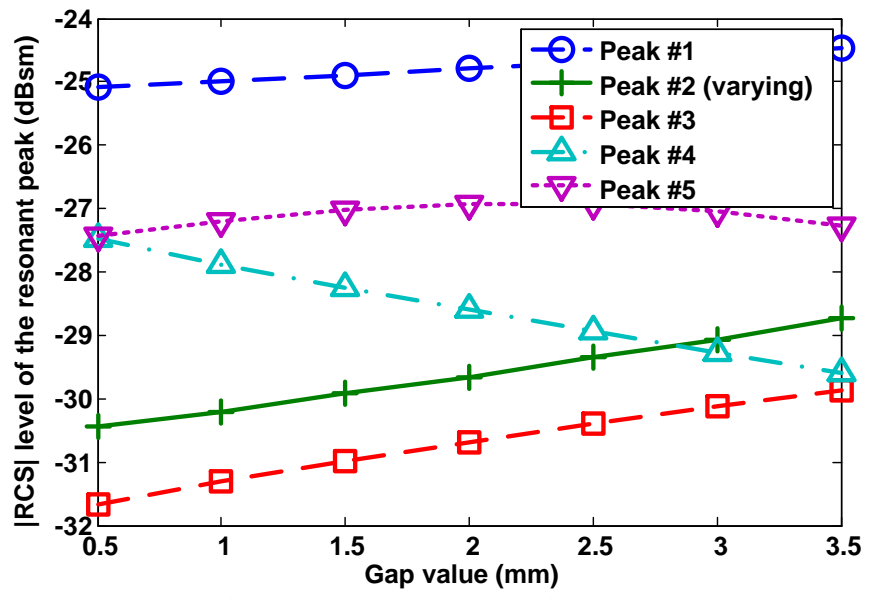

Fig. 8. Variation of the RCS magnitude level of the peaks in the surrounded configuration when only the gap value of the second C-folded dipole is varied. The variation shows an important coupling between the scatterers.

implementations [22], [23], could achieve higher capacity gain. Considering the hypothetical case where the magnitude of each resonator can be set independently with a comparable magnitude range would lead to: $\ln _{2}\left(6^{5} \times 4^{5}\right)=13+10$ bits which illustrate the potential gain of the magnitude coding. This type of coding would be particularly interesting for tag having lossy substrates (like paper) where the FP coding has limited efficiency. This coding can be straightforwardly applied to the tags previously studied in [11], [17] (same geometry than the present article) with no additional complexity for the design or at the reader part.

In the following, we consider that the current design is sufficient to assess the feasibility of the magnitude coding approach. The possibility to effectively retrieve the magnitude information in practice still needs to be demonstrated. The minimum resolution that can be measured has to be evaluated. The significant magnitude span of the first three peaks will allow an evaluation on a frequency band from 2.5 to $4.5 \mathrm{GHz}$.

\section{LeVel Magnitude Coding FeasibiLity}

The RCS magnitude level is known to be particularly sensitive to perturbations. We are seeking to evaluate in practice the minimum variation of the RCS that can be detected in measurement. For that, different factors that can modify the RCS magnitude of the tag are investigated. The tags designed in the previous section are measured in anechoic chamber at various distances to estimate the measurement accuracy. The influence of the tagged object is then considered as well as the eventual presence of an obstacle between the tag and the reader. Finally the tag is measured in real environment.

\section{A. Measurement accuracy with respect to distance}

The theoretical definition of the RCS does not depend on the distance between tag and antenna [27]. However, in practice, the measured quantity is the backscattered power received at the antenna, which depends strongly on the measurement setup. A usual way to obtain the exact value of $\sigma$ is to proceed to a reference calibration measurement, as done in [17]. In this article, a measurement setup similar to the one in [17] is done (Fig. 9). Measurements are done in the frequency domain with the Agilent PNA Network Analyzer N5222A in bistatic configuration for the vertical polarization. The power delivered 


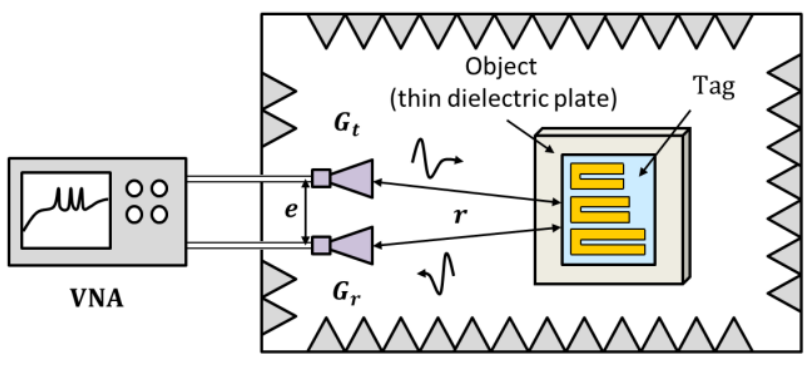

Fig. 9. Measurement Setup. Bistatic configuration. The measurement is done in an anechoic chamber.

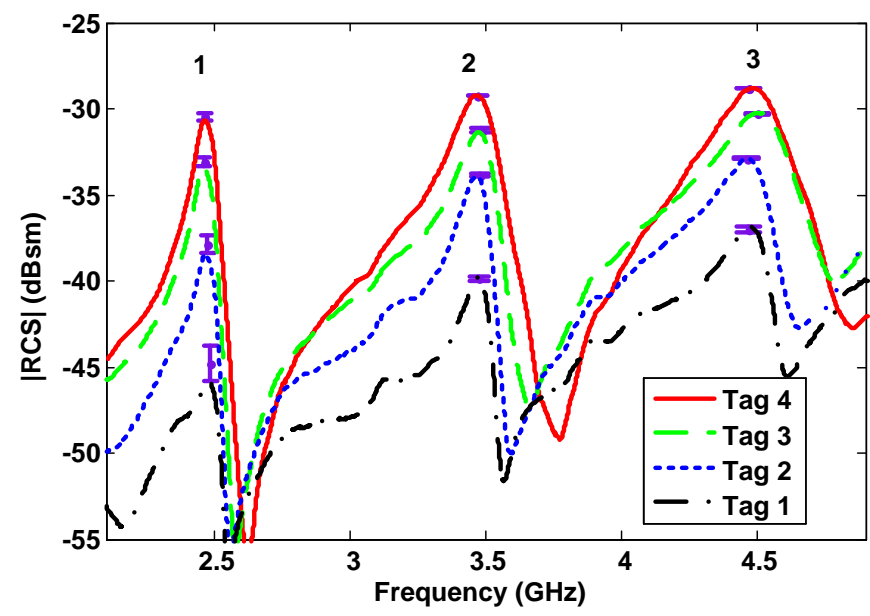

Fig. 10. RCS magnitude level of the three first peaks measured at $60 \mathrm{~cm}$ Peaks levels obtained by measurement at $70 \mathrm{~cm}$ and $80 \mathrm{~cm}$ are indicated by the lower and upper bound of the error bars, respectively. Maximum deviation of $2.1 \mathrm{~dB}$ is observed for the first peak of tag 1 .

by the vector network analyzer (VNA) is $0 \mathrm{dBm}$ in the frequency band from $2 \mathrm{GHz}$ to $8 \mathrm{GHz}$.The two horn antennas have a gain of $12 \mathrm{dBi}$ in the frequency band of interest. The spacing between the antennas is $e=30 \mathrm{~cm}$. The measured quantities correspond to the $S_{21}$ parameter. The tag is placed at a minimum distance of $r=60 \mathrm{~cm}$ from both antennas. An isolation measurement with no tag $\left(S^{\text {isolation }}\right)$ and a reference measurement $\left(S^{r e f}\right)$ with a metallic plate with a known RCS $\left(\sigma^{r e f}\right)$ are done. This allows extracting the exact value of the RCS using (7):

$$
\sigma^{\text {tag }}=\left[\frac{s^{\text {tag }}-S^{\text {isolation }}}{S^{r e f}-S^{\text {isolation }}}\right]^{2} \cdot \sigma^{r e f},
$$

where $\left(S^{\text {tag }}\right)$ is the measured $S$ parameter and $\sigma^{\text {tag }}$ is the extracted value of the complex RCS corresponding to the measured tag.

The curves shown Fig. 10 correspond to the RCS calculated with (7) for the tags 1-4 positioned at a distance $r=60 \mathrm{~cm}$. The RCS extraction was also performed from measurement of tags positioned at $70 \mathrm{~cm}$ and $80 \mathrm{~cm}$. The peak magnitude deviations are represented in Fig. 10 by error bars. The lower and upper bounds correspond to the magnitude of the peak at $70 \mathrm{~cm}$ and $80 \mathrm{~cm}$, respectively. A maximum deviation of $2.1 \mathrm{~dB}$ is obtained for the first peak of the tag 1 , which corresponds to the lowest magnitude level $(-45 \mathrm{~dB})$, and is then more sensitive to noise. In other cases, the deviation is less than $1.4 \mathrm{~dB}$. The magnitude resolution in $\mathrm{dB}$ has to be superior to $\Delta \sigma=2.1 \mathrm{~dB}$ to be able to discriminate the level of each peak for a read range of $80 \mathrm{~cm}$. When increasing the distance, the power backscattered to the antenna becomes lower due to free space path loss and the signal-to-noise ratio decreases. For a larger reading distance, a higher magnitude resolution would certainly be necessary.

\section{B. Tag applied on unknown object}

In a practical case, the tag is adhered to the surface of an unknown object and the total backscattered field $\overrightarrow{\boldsymbol{E}_{t}}(f)$ is the sum of two components: $\left|\overrightarrow{\boldsymbol{E}_{t}}\right|=\left|\overrightarrow{\boldsymbol{E}_{r}}+\overrightarrow{\boldsymbol{E}_{o}}\right|$ where $\overrightarrow{\boldsymbol{E}_{r}}(f)$ is the response of the tag and $\overrightarrow{\boldsymbol{E}_{o}}(f)$ corresponds to the specular reflection on the object. We will consider in a first time that there is no interaction between the tag and the object; that is, a variation of the object characteristics modifies only $\overrightarrow{\boldsymbol{E}_{o}}(f)$ without affecting $\overrightarrow{\boldsymbol{E}_{\boldsymbol{r}}}(f)$. The effects of the interaction will be discussed in a second step.

The root of the total $\operatorname{RCS} \sqrt{\sigma}$ is related to the ratio between the total backscattered electric field $\overrightarrow{\boldsymbol{E}_{\boldsymbol{t}}}$ and the incident electric field $\overrightarrow{\boldsymbol{E}_{\iota}}$ by (8) [27]:

$$
\sqrt{\sigma}=\lim _{r \rightarrow \infty} 2 \sqrt{\pi} r \frac{\left|\overrightarrow{\boldsymbol{E}_{t}}\right|}{\left|\overrightarrow{\boldsymbol{E}_{l}}\right|} .
$$

In far-field, (8) can be rewritten as $\sqrt{\sigma}=A\left|\overrightarrow{\boldsymbol{E}_{t}}\right|$, where $A$ is a normalization constant with respect to the distance and the incoming field. The object contribution can be considered as a random component affecting the magnitude level of the RCS which can lead to bit errors. To remove this contribution, a relative measurement can be done instead of directly comparing the RCS values. Let consider two different tags, for instance, tag 2 and tag 4 (indicated by the subscripts 2 and 4 respectively). Those tags are successively applied on the same object and the corresponding RCS is measured. The quantity that remains constant regardless of the object is the difference between the total re-radiated fields (9):

$$
\Delta E=\left|\overrightarrow{\boldsymbol{E}_{t 4}}-\overrightarrow{\boldsymbol{E}_{t 2}}\right|=\left|\overrightarrow{\boldsymbol{E}_{r 4}}-\overrightarrow{\boldsymbol{E}_{r 2}}\right|,
$$

with no dependence on $\overrightarrow{\boldsymbol{E}_{o}}$. Considering the peak apex, the annulation of the phase at the resonance allows to express $\triangle E$ in function of the RCS:

$$
\begin{aligned}
A \cdot \Delta E & =A \cdot\left|\overrightarrow{\boldsymbol{E}_{t 4}}-\overrightarrow{\boldsymbol{E}_{t 2}}\right|=A \cdot\left|\overrightarrow{\boldsymbol{E}_{t 4}}\right|-A \cdot\left|\overrightarrow{\boldsymbol{E}_{t 2}}\right| \\
& =\sqrt{\sigma_{4}}-\sqrt{\sigma_{2}},
\end{aligned}
$$

which does not depend on distance or on incoming power as $\sigma_{4}$ and $\sigma_{2}$ are normalized values.

For tags having no ground planes, the interaction between the tag and the object may modify significantly the response of the tag itself $\left(\overrightarrow{\boldsymbol{E}_{r}}\right.$ in the previous equations). Two main effects can be expected. First the object will modify the effective permittivity of the tag. If the permittivity and the width of the object are known, the effective permittivity $\varepsilon_{e}{ }^{\prime}$ associated to the resonator can be calculated analytically similar to CPS technology with multilayer substrate [24]. Using $\varepsilon_{e}{ }^{\prime}$ in (1) shows that the presence of the object introduces a deviation of the resonant frequency 


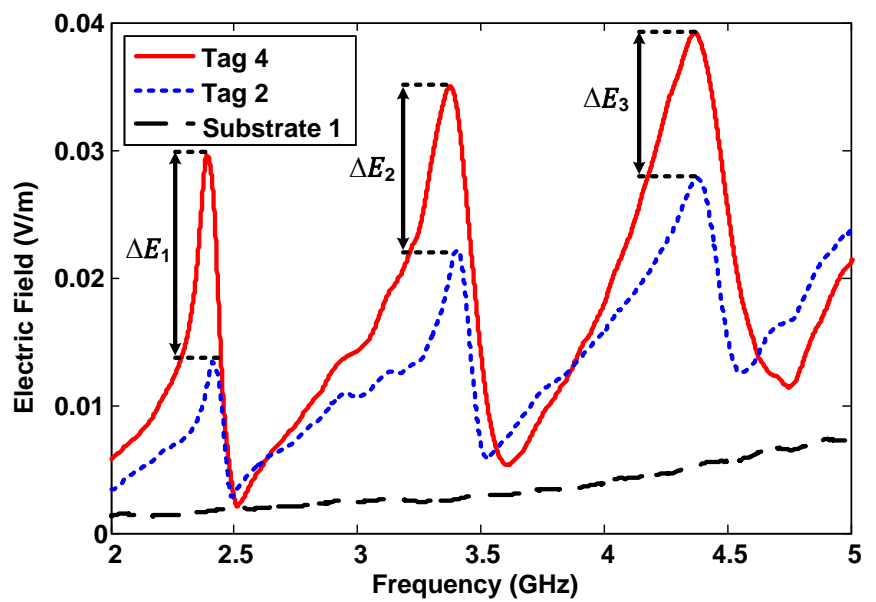

(a)

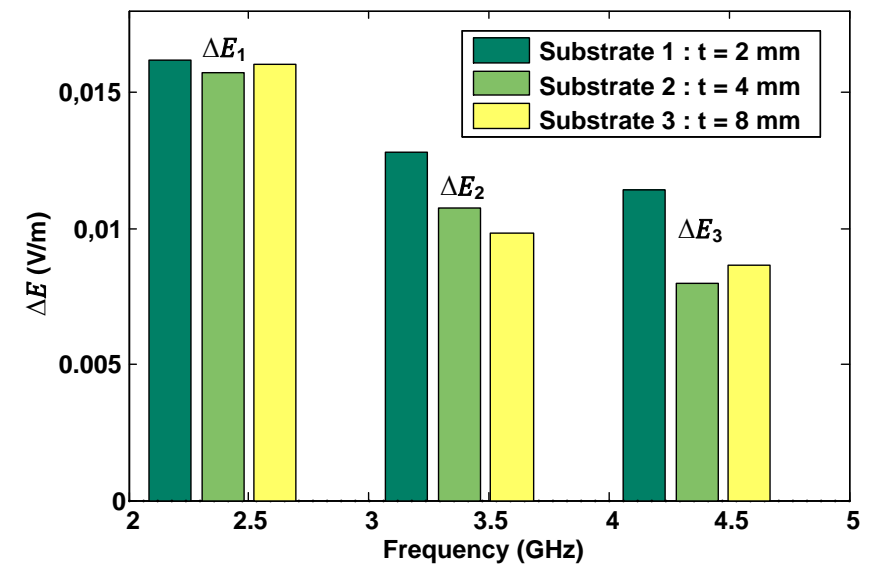

(b)

Fig. 11. (a) Re-radiated field difference for tag 2 and 4 applied on the first dielectric plate. (b) Variation of the re-radiated field difference with respect to the substrate for the three peaks.

corresponding to the coefficient $\sqrt{\varepsilon_{e}} / \sqrt{\varepsilon_{e}{ }^{\prime}}$. This phenomenon has been studied in [11] and a compensation technique has been proposed. Secondly, the object introduces additional losses which can modify the quality factor of the resonators as it is expected from (6). Other pattern change can arise due to the modification of the couplings between the unit scatterers but are more difficult to characterize. A sufficient resolution has to be determined to account for those eventual effects.

To verify that $\Delta E$ remains nearly constant, measurements with different objects have been carried out. The objects constituted of a dielectric plate of thicknesses $t=2 \mathrm{~mm}, 4 \mathrm{~mm}$, $8 \mathrm{~mm}$, and $12 \mathrm{~mm}$ numbered from 1 to 4 , respectively. The plate dimensions are $45 \mathrm{~mm} \times 55 \mathrm{~mm}$ and the relative permittivity of the dielectric is 3 . Tags 2 and 4 are successively applied on the top of the objects and the corresponding RCS is measured. The presence of multiple scatterers within the tags allows verifying the assumption for a frequency band from $2.5 \mathrm{GHz}$ to $4.5 \mathrm{GHz}$ and different magnitude values.

Figure 12-a shows the electric field response of the tags 2 and 4 positioned on the substrate 1 . For the $i^{\text {th }}$ peak, the value $\Delta E_{i}$ is the difference between the re-radiated fields of the two tags. The values of $\Delta E_{i}$ are compared for the three substrates in Fig. 12-b. The value is nearly constant for the first peak. A variation of 0.2 and 0.3 of the full scale is obtained for the peaks 2 and 3. This can be because of either measurement inaccuracy or the interaction between the object and the tag that has been neglected. The relative variation can be expressed in $\mathrm{dB}(3.1 \mathrm{~dB}$ and $1.9 \mathrm{~dB}$ for the peaks 2 and 3, respectively) to determine an equivalent magnitude resolution in $\mathrm{dB}$, which takes into account the presence of the object. The necessary resolution is considered to be $3.5 \mathrm{~dB}$. It is superior to the $2.1 \mathrm{~dB}$ resolution obtained in the last section, which is logical if we consider the object as an additional noise. Following this analogy, if we consider an object with a larger RCS value, the signal-to-noise ratio will decrease and a higher magnitude resolution would certainly be necessary. If the object contribution is too important compared to the RCS of the tag, the signal-to-noise ratio will be close to zero and no tag measurement will be possible. In this case a prior calibration of the object may be necessary. The limit case can be considered when the RCS of the object is equal to the RCS value of the object.

For small objects (RCS values comparable to the one in the dielectric plates described in this section), a resolution of $3.5 \mathrm{~dB}$ is considered sufficient to discriminate the different magnitude levels. As the magnitude range of the design tags is $15.1 \mathrm{~dB}$, four different levels can be set, which correspond to an additional coding capacity of two bits.

\section{Coated Tag}

An interesting case of application is a tagged object placed inside of a carton box. In this case, no direct line of sight exists and the obstacle between the tag and the reader attenuates the signal which can lead to bit error. Similarly to the previous section, a relative measurement can be done to prevent errors. Measurements of tags coated by two different dielectrics sheets (denoted by 1 and 2) of thickness $t_{1}=0.83$ $\mathrm{mm}$ and $t_{2}=0.68 \mathrm{~mm}$, permittivity $\varepsilon_{1}=5.1$ and $\varepsilon_{2}=2.7$ and loss tangent $\delta_{1}=0.18$ and $\delta_{2}=0.02$ have been performed. Distance between tag and reader is $60 \mathrm{~cm}$ and the dielectric sheets have been applied directly on the tag. The measurement results are presented in Fig. 12. Similar behavior than for a tag applied on a dielectric object are observed. The change of permittivity due to the presence of the dielectric sheets in the vicinity of the tag induces a frequency deviation of $450 \mathrm{MHz}$ and $140 \mathrm{MHz}$ for dielectric 1 and 2 respectively. If the sheets are not touching the tag, no frequency deviation is observed. The quality factor of the resonance is lower for dielectric sheet 1 due to higher loss. Like in the previous section, the relative quantity corresponding to $\Delta E$ remains nearly constant if the dielectric has a low loss (see comparison between no sheet and dielectric sheet 2 in Table III). When the dielectric sheet has higher loss (dielectric sheet 1), the relative measure varies in a similar way for all peaks (factor 0.5 when comparing no sheet with dielectric sheet 1 in Table III). The resolution of $3.5 \mathrm{~dB}$ which has been considered in the previous section is sufficient in regards of the variation of $\Delta \mathrm{E}$. Like in the previous section the limit case can be considered when the

TABLE III

RELATIVE MEASUREMENT OF COATED TAGS

\begin{tabular}{|l|c|c|c|}
\hline & $\Delta E_{1}(\mathrm{~V} / \mathrm{m})$ & $\Delta E_{2}(\mathrm{~V} / \mathrm{m})$ & $\Delta E_{3}(\mathrm{~V} / \mathrm{m})$ \\
\hline No sheet & 0.010 & 0.008 & 0.006 \\
\hline Dielectric sheet 1 & 0.005 & 0.004 & 0.003 \\
\hline Dielectric sheet 2 & 0.011 & 0.009 & 0.005 \\
\hline
\end{tabular}




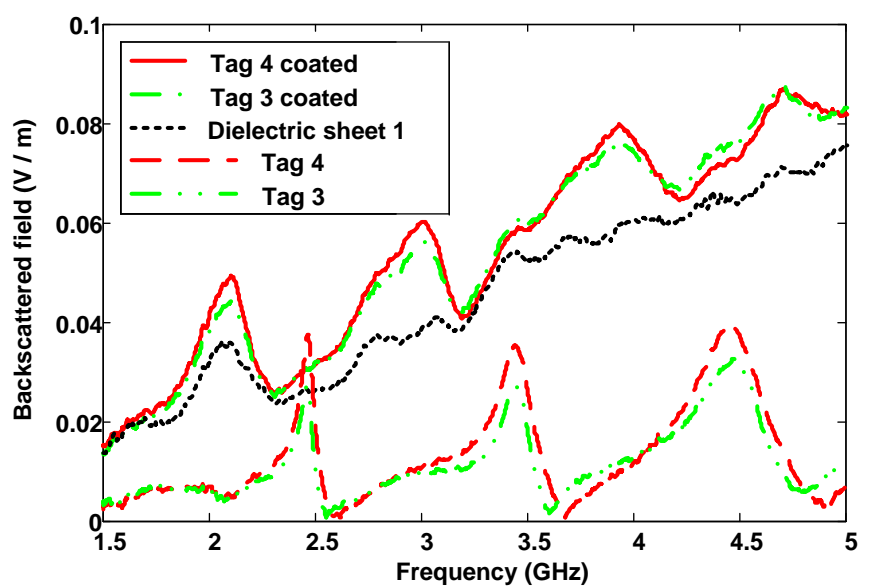

(a)

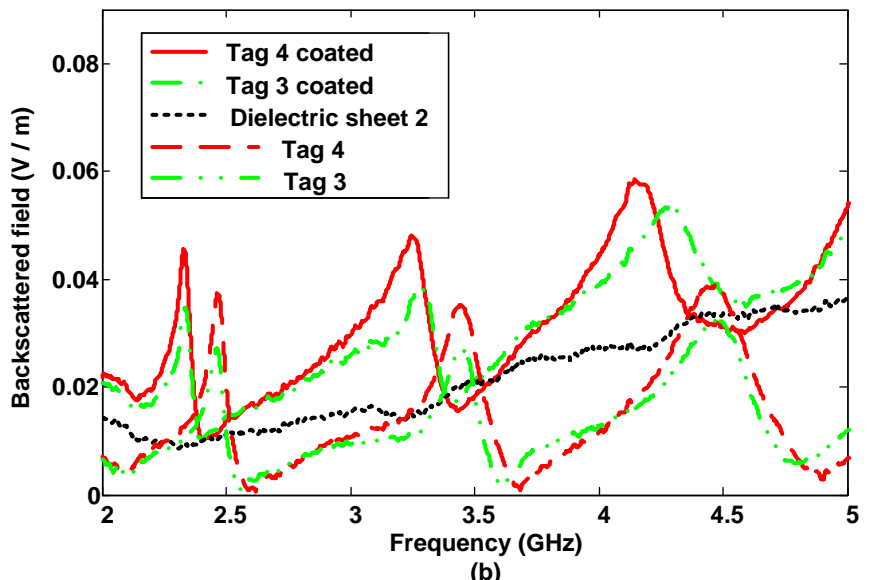

Fig. 12. Relative measurement of coated tags (a) Re-radiated field for tag 3 and 4 coated by a dielectric sheet of thickness $t_{1}=0.83 \mathrm{~mm}$, permittivity $\varepsilon_{1}=5.1$ and loss tangent $\delta_{1}=0.18$. (b) Re-radiated field for tag 3 and 4 coated by a dielectric sheet $t_{2}=0.68 \mathrm{~mm}, \varepsilon_{2}=2.7$ and $\delta_{1}=0.02$

RCS of the dielectric sheet is higher than the RCS of the tag itself (Fig. 12. a).

\section{Real environment measurement}

The tags have been measured in an office environment. The distance tag-reader has been decreased to $20 \mathrm{~cm}$ because of the higher noise level. To ensure far-field condition at this distance, the horn antennas used in the anechoic chamber configuration have been replaced by Satimo QH2000 antennas in bistatic configuration. These antennas present a gain of $3 \mathrm{dBi}$ to $8 \mathrm{dBi}$ in the frequency band $2 \mathrm{GHz}$ to $5 \mathrm{GHz}$. A photograph of the measurement setup is given in Fig. 13.

The tags 1-4 have been measured and their response is presented on Fig. 14. A rectangular time window has been applied to the measured data to limit the impact of the reflection on the wall visible on Fig. 13. The magnitude levels of the second and third peak of tags 1-4 are similar to the anechoic chamber results (Fig. 7). For instance, considering the second peak at $3.5 \mathrm{GHz}$ of Tag 4, a RCS value of $-28 \mathrm{dBsm}$ is obtained in real environment compared to $-28.5 \mathrm{dBsm}$ in anechoic configuration. For all the tags, the first peak present lower magnitude level than the anechoic configuration. This difference can be explained both by the interference of the $\mathrm{Wi}-\mathrm{Fi}$ at $2.4 \mathrm{GHz}$ and to the lower gain of the antennas at this frequency. Nevertheless, the distance

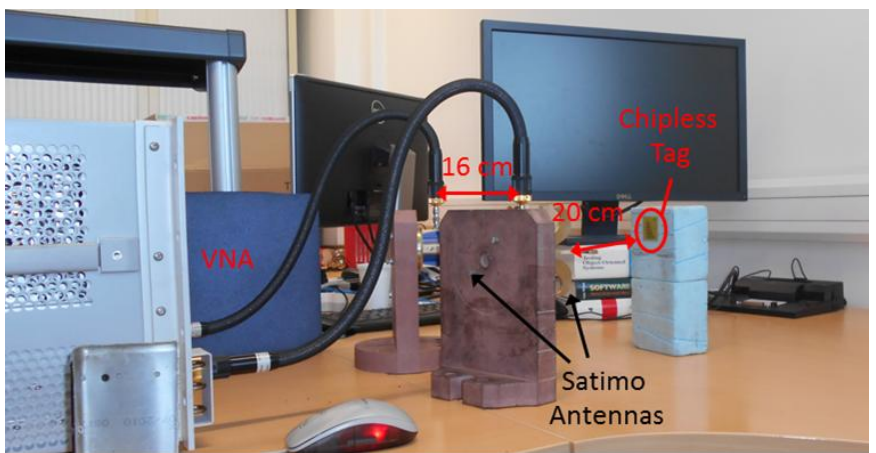

Fig. 13. Real environment measurement setup. The distance between tag and antennas is $20 \mathrm{~cm}$. The antennas are Satimo QH2000 to ensure far-field condition at this distance.

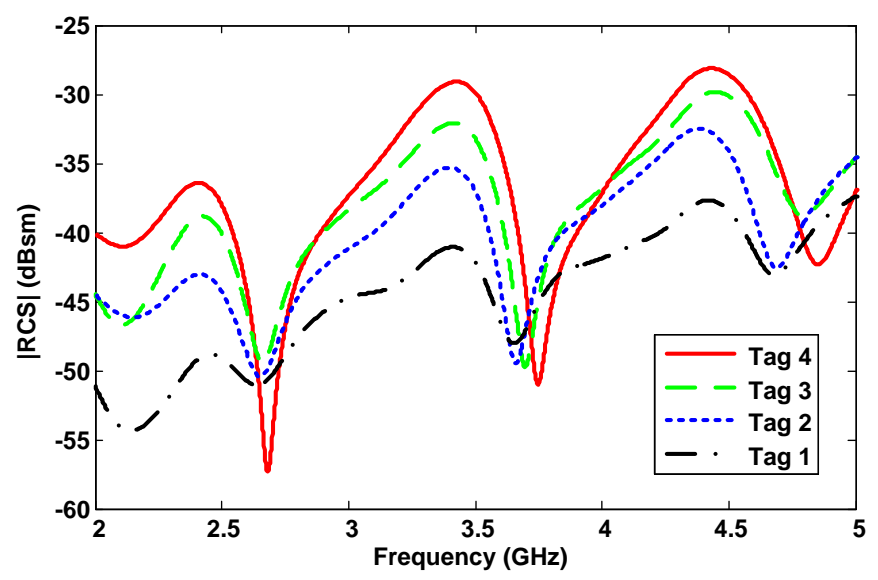

Fig. 14. RCS measurement of the tags in real environment. A time window has been applied to remove the reflection of the wall.

between the different levels remains similar than in anechoic configuration ( $2.1 \mathrm{dBsm}$ between the first peak of tags 4 and 3 in real environment compared to $2.3 \mathrm{dBsm}$ in anechoic configuration). The frequency position of the peaks remains identical for both configurations. The selectivity of the peaks is less important than in anechoic configuration due to the additional noise.

The measurement realized in real environment gives results comparable to the anechoic configuration. The main difference is the lower read range that can be achieved. When the tags are readable, the resolution of $3.5 \mathrm{dBsm}$ assessed from anechoic measurement can be considered sufficient even for real environment applications since no critical degradation of the signal is observed.

\section{COMPENSATION TECHNIQUE}

For a practical use, it is necessary to be able to determine the magnitude code from one tag alone. If the object is unknown, the augmentation of the RCS magnitude level because of the object contribution can be misinterpreted as a different coding than expected. Example of such a case is represented by measurements in Fig. 15. Tag 2 and tag 4 are supposed to code different information. If they are applied on the substrate 1 and 4 respectively, we can see that the magnitude of the second peak is the same for both configurations and it is then impossible to differentiate the tags. Additionally a detuning of the frequency is observed. 


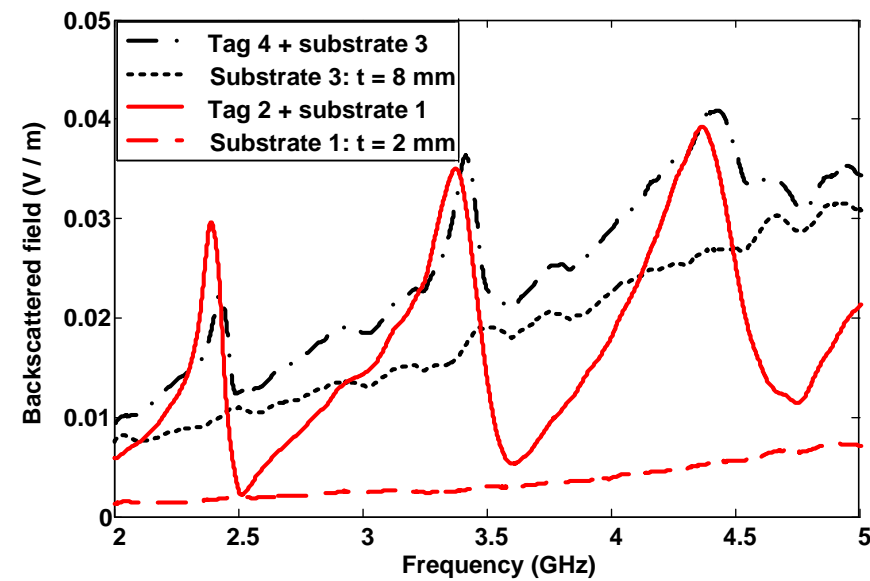

Fig. 15. Level detection error because of unknown object contribution. The magnitude value of the second peak is the same for tag 2 and tag 4 .

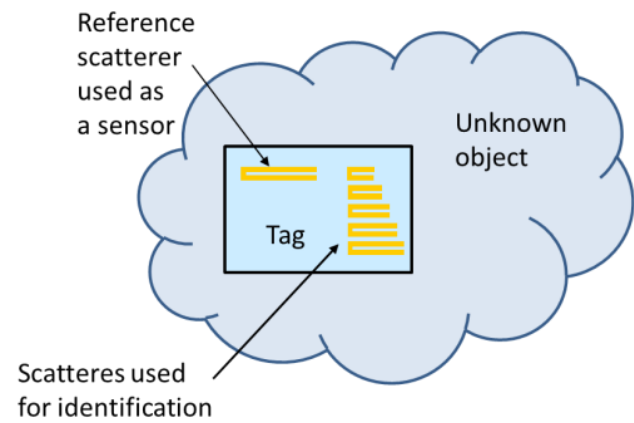

Fig. 16. Compensation principle based on a reference scatterer. The additional scatterer has a known RCS level in absence of object. It is used to "sense" the RCS magnitude of

A compensation method has been proposed for FP coding in [11] to compensate the detuning of the frequency but with no consideration for the magnitude level. This method can be adapted to recover the magnitude code as if the measurement was made in free space (absence of object). The method applies for objects a having low RCS value and for which a model is available (like thin dielectric substrate). The limit case can be considered as object having RCS higher than the tag itself.

As in the previous section, a relative measurement can be done. But this time, the reference measurement is included directly within the tag in the form of an additional reference scatterer (Fig. 16). The magnitude level of the reference scatterer is supposed to be known in absence of object. When the tag is applied on the object, the variation of the magnitude value of the reference scatterer can be used to sense the object contribution. The reference scatterer has to be spaced far enough from the other scatterers to avoid parasitic couplings of tags having different codes.

As the reference scatterer is a resonator, it provides information only at its resonant frequency but not for the whole band of interest. A model of the object RCS has then to be established to recover the information on the whole band. If the object can be assimilated to a thin dielectric plate, a closed form formula based on dielectric physical optic has been derived in [28] with very good accuracy:

$$
\sqrt{\sigma_{s}}=\pi \sqrt{4 \pi} \frac{t(\varepsilon-1) S}{c^{2}} f^{2} .
$$

where $t, \varepsilon$ and $S$ are the thickness, permittivity and surface of the dielectric.

We consider that the magnitude level and resonant frequency of the reference scatterer $\overrightarrow{\boldsymbol{E}_{r}}\left(f_{0}\right)$ are known in absence of object. When an object is introduced, the measured value of the peak is modified:

$$
\overrightarrow{\boldsymbol{E}_{m}}\left(f_{0}\right)=\overrightarrow{\boldsymbol{E}_{r}}\left(f_{0}\right)+\overrightarrow{\boldsymbol{E}_{o}}\left(f_{0}\right) \text {. }
$$

If the object can be assimilated to a thin dielectric, (11) gives:

$$
\left|\overrightarrow{\boldsymbol{E}_{m}}\left(f_{0}\right)-\overrightarrow{\boldsymbol{E}_{\boldsymbol{r}}}\left(f_{0}\right)\right|=\left|\overrightarrow{\boldsymbol{E}_{o}}\left(f_{0}\right)\right|=A \pi \sqrt{4 \pi} \frac{t(\varepsilon-1) S}{c^{2}} f_{0}^{2},
$$

The response of the object in the whole frequency band can be deduced from (11)-(13):

$$
\overrightarrow{\boldsymbol{E}_{o}}(f)=\left[\overrightarrow{\boldsymbol{E}_{m}}\left(f_{0}\right)-\overrightarrow{\boldsymbol{E}_{r}}\left(f_{0}\right)\right]\left(\frac{f}{f_{0}}\right)^{2} .
$$

Once the response of the substrate is reconstructed, it is possible to extract the expected RCS magnitude level of the tag from (12). The reference value in absence of object $\overrightarrow{\boldsymbol{E}_{r}}\left(f_{0}\right)$ can be obtained either in measurement like in Fig. 17, or by simulation.

A validation of the compensation method has been done with measurement. The tag 2 has been measured for three configurations: in absence of object, applied on substrate 1,

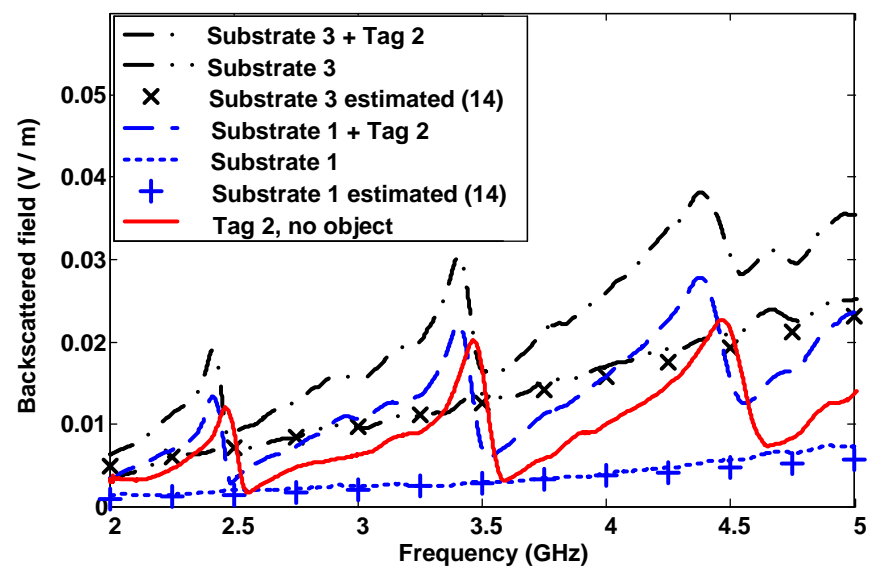

Fig. 17. Evaluation of the signature of the unknown substrates (thin dielectric) using a reference scatterer (measurements). The first peak of the configuration without object is considered as the reference value.

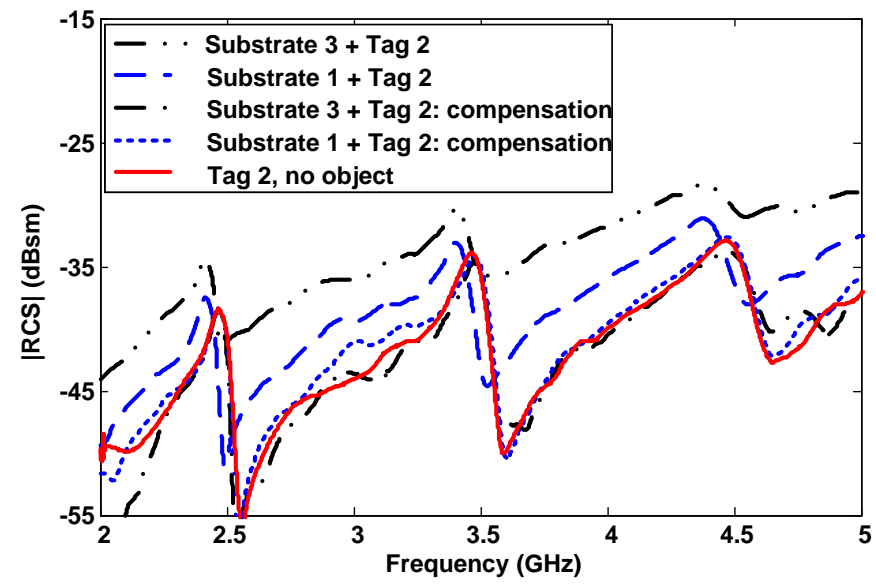

Fig. 18. Comparison of the signature of tag 2 obtained by compensation method with the signature in absence of object (measurements). 
and applied on substrate 3 (Fig. 17). As expected, the tag shows different magnitude levels in presence of substrate. An additional frequency shift of approximately $100 \mathrm{MHz}$ because of the interaction between the substrate and the tag can be observed. The first peak of the tag 2 in absence of object (red line) is used as the reference scatterer. The substrate signatures on the whole frequency band are calculated using (14) and are compared with the measurement for both substrates on Fig. 17 with good agreement. Following the evaluation of the substrate response, the tag response without object can be recovered by subtraction of the fields. Additional frequency compensation is done as in [11] to obtain the correct frequency positions of the peaks. The compensated signatures are shown in Fig. 18 and are in good agreement with the measurement of the configuration without object. A maximum deviation of $1.6 \mathrm{~dB}$ is observed for the third peak of the substrate 3 configuration.

\section{CONCLUSION}

The possibility to code information with the magnitude has been evaluated in practice. A model taking into account the radiation properties of the $\mathrm{C}$-like resonators has been used to design four tags with different magnitude levels. A magnitude span of up to $15.2 \mathrm{~dB}$ has been obtained for coupled resonators. A minimum resolution of $3.5 \mathrm{~dB}$ has been evaluated from measurements providing an additional coding of 2 bits for the current design. The problem of the presence of an unknown object has been addressed and a compensation technique has been proposed for objects similar to thin dielectric plates.

The magnitude coding technique can be of interest for tags printed on paper. In this case the quality factors of the resonators are much lower due to the important loss, which limits the efficiency of the FP coding. For tags printed on paper, a specific design has to be done with a particular attention to the RCS level and the selectivity of the resonators. The control of the RCS level is then an underlying problematic for the design of tags printed on paper which could be exploited advantageously with magnitude coding.

\section{ACKNOWLEDGMENTS}

The authors would like to thank Dr. A. Vena for the design and realization of the tags presented in this article.

\section{REFERENCES}

[1] E. Perret, Radio Frequency Identification and Sensors: From RFID to Chipless RFID. John Wiley \& Sons, 2014.

[2] S. Preradovic, I. Balbin, N. C. Karmakar, and G. F. Swiegers, "Multiresonator-Based Chipless RFID System for Low-Cost Item Tracking," IEEE Trans. Microw. Theory Techn., vol. 57, no. 5, pp. 1411-1419, May 2009.

[3] A. Vena, E. Perret, S. Tedjini, G. E. P. Tourtollet, A. Delattre, F. Garet, and Y. Boutant, "Design of Chipless RFID Tags Printed on Paper by Flexography," IEEE Trans. Antennas Propag., vol. 61, no. 12, pp. 5868-5877, Dec. 2013.

[4] S. Preradovic and N. C. Karmakar, "Chipless RFID: Bar Code of the Future," IEEE Microw. Mag., vol. 11, no. 7, pp. 87-97, Dec. 2010.

[5] S. Tedjini, N. Karmakar, E. Perret, A. Vena, R. Koswatta, and R. EAzim, "Hold the Chips: Chipless Technology, an Alternative Technique for RFID," IEEE Microw. Mag., vol. 14, no. 5, pp. 56-65, Jul. 2013.
[6] D. Girbau, A. Ramos, A. Lazaro, S. Rima, and R. Villarino, "Passive Wireless Temperature Sensor Based on Time-Coded UWB Chipless RFID Tags," IEEE Trans. Microw. Theory Techn., vol. 60, no. 11, pp. 3623-3632, Nov. 2012.

[7] C. S. Hartmann, "A global SAW ID tag with large data capacity," in 2002 IEEE Ultrasonics Symposium, 2002. Proceedings, 2002, vol. 1, pp. 65-69 vol.1.

[8] B. Shao, Q. Chen, Y. Amin, D. S. Mendoza, R. Liu, and L.-R. Zheng, "An ultra-low-cost RFID tag with 1.67 Gbps data rate by ink-jet printing on paper substrate," in Solid State Circuits Conference (ASSCC), 2010 IEEE Asian, 2010, pp. 1-4.

[9] A. Vena, E. Perret, and S. Tedjini, "Design rules for chipless RFID tags based on multiple scatterers," Ann. Telecommun.-Ann. Télécommunications, vol. 68, no. 7-8, pp. 361-374, 2013.

[10] R. Rezaiesarlak and M. Manteghi, "Complex-Natural-ResonanceBased Design of Chipless RFID Tag for High-Density Data," IEEE Trans. Antennas Propag., vol. 62, no. 2, pp. 898-904, Feb. 2014.

[11] A. Vena, E. Perret, and S. Tedjini, "A Fully Printable Chipless RFID Tag With Detuning Correction Technique," IEEE Microw. Wirel. Compon. Lett., vol. 22, no. 4, pp. 209-211, Apr. 2012.

[12] S. Preradovic and N. C. Karmakar, "Design of Chipless RFID Tag for Operation on Flexible Laminates," IEEE Antennas Wirel. Propag. Lett., vol. 9, pp. 207-210, 2010.

[13] D. Girbau, J. Lorenzo, A. Lazaro, C. Ferrater, and R. Villarino, "Frequency-Coded Chipless RFID Tag Based on Dual-Band Resonators," IEEE Antennas Wirel. Propag. Lett., vol. 11, pp. 126$128,2012$.

[14] C. M. Nijas, U. Deepak, P. V. Vinesh, R. Sujith, S. Mridula, K. Vasudevan, and P. Mohanan, "Low-Cost Multiple-Bit Encoded Chipless RFID Tag Using Stepped Impedance Resonator," IEEE Trans. Antennas Propag., vol. 62, no. 9, pp. 4762-4770, Sep. 2014.

[15] M. A. Islam and N. C. Karmakar, "A Novel Compact Printable DualPolarized Chipless RFID System," IEEE Trans. Microw. Theory Techn., vol. 60, no. 7, pp. 2142-2151, Jul. 2012.

[16] M. S. Bhuiyan and N. Karmakar, "A spectrally efficient chipless RFID tag based on split-wheel resonator," in 2014 International Workshop on Antenna Technology: "Small Antennas, Novel EM Structures and Materials, and Applications" (iWAT), 2014, pp. 1-4.

[17] A. Vena, E. Perret, and S. Tedjini, "Chipless RFID Tag Using Hybrid Coding Technique," IEEE Trans. Microw. Theory Techn., vol. 59, no. 12, pp. 3356-3364, Dec. 2011.

[18] S. Gupta, B. Nikfal, and C. Caloz, "Chipless RFID System Based on Group Delay Engineered Dispersive Delay Structures," IEEE Antennas Wirel. Propag. Lett., vol. 10, pp. 1366-1368, 2011.

[19] R. Nair, E. Perret, and S. Tedjini, "Chipless RFID based on group delay encoding," in 2011 IEEE International Conference on RFIDTechnologies and Applications (RFID-TA), 2011, pp. 214-218.

[20] C. Feng, W. Zhang, L. Li, L. Han, X. Chen, and R. Ma, "Angle-Based Chipless RFID Tag With High Capacity and Insensitivity to Polarization," IEEE Trans. Antennas Propag., vol. 63, no. 4, pp. 1789-1797, Apr. 2015.

[21] A. El-Awamry, M. Khaliel, A. Fawky, M. El-Hadidy, and T. Kaiser, "Novel notch modulation algorithm for enhancing the chipless RFID tags coding capacity," in 2015 IEEE International Conference on RFID (RFID), 2015, pp. 25-31.

[22] A. Vena, A. A. Babar, L. Sydanheimo, M. M. Tentzeris, and L. Ukkonen, "A Novel Near-Transparent ASK-Reconfigurable InkjetPrinted Chipless RFID Tag," IEEE Antennas Wirel. Propag. Lett., vol. 12, pp. 753-756, 2013.

[23] O. Rance, R. Siragusa, P. Lemaitre-Auger, and E. Perret, "RCS magnitude coding for chipless RFID based on depolarizing tag," in Microwave Symposium (IMS), 2015 IEEE MTT-S International, 2015, pp. $1-4$.

[24] R. Garg, I. Bahl, and M. Bozzi, Microstrip lines and slotlines. Artech house, 2013.

[25] S. Gov and S. Shtrikman, "On isotropic scattering of electromagnetic radiation," in , Eighteenth Convention of Electrical and Electronics Engineers in Israel, 1995, 1995, pp. 2.4.4/1-2.4.4/5.

[26] J. Machac and M. Polivka, "Influence of mutual coupling on performance of small scatterers for chipless RFID tags," in Radioelektronika (RADIOELEKTRONIKA), 2014 24th International Conference, 2014, pp. 1-4.

[27] E. F. Knott, J. F. Shaeffer, and M. T. Tuley, Radar Cross Section. Dedham, MA: Artech House. Inc, 1985. 
[28] D. M. Le Vine, A. Schneider, R. H. Lang, and H. Carter, "Scattering from thin dielectric disks," IEEE Trans. Antennas Propag., vol. 33, no. 12, pp. 1410-1413, Dec. 1985.

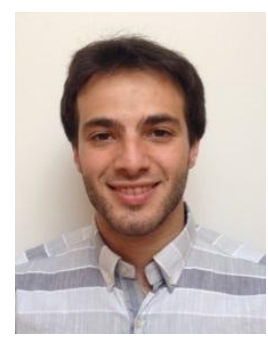

Olivier Rance (S'15) is a $\mathrm{PhD}$ student working under the supervision of Dr. Etienne Perret. He received his Master's degree in Electronic Engineering from the Institute National Polytechnique de Grenoble (Grenoble-INP), and joined the LCIS in 2012. His main research interests are leaky-wave antennas and chipless RFID.

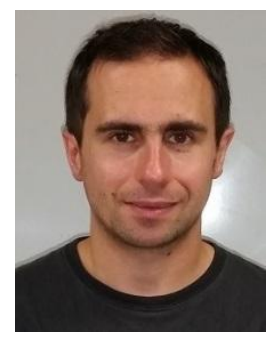

Romain Siragusa was born in Paris, France. He received the engineer degree and the master degree from the Institute National Polytechnique de Grenoble (Grenoble-INP) in 2006 and the Ph.D degree from the same institute at the Laboratoire de Conception et d'Intégration des Systèmes (LCIS) in Valence, France. In 2010/2009, he joined the "commissariat à l'énergie atomique" (CEA) as post doctoral fellow where he studied the high impedance surface for integrated antenna on silicon. Since 2011, he is associate professor at Grenoble-INP Esisar / LCIS. His current scientific interests include leakywave structures and Chipless RFID.

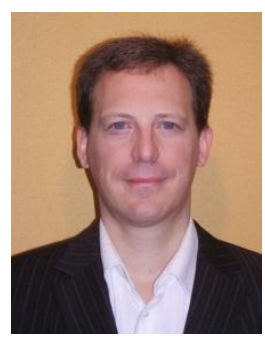

Pierre Lemaitre-Auger received the B. Ing. and the M. Sc. A. degrees from the École Polytechnique de Montréal, Canada, in 1992 and 1994 respectively in physical engineering. He obtained his doctor degree in Optoelectronics and Microwave from the Institut National Polytechnique de Grenoble, France, in 1998. He joined the engineering school Esisar (Valence, France) of the Institut Polytechnique de Grenoble and the LCIS Laboratory in 1998 as an associate professor He participated to the creation of the Esisar and especially to the elaboration of its physics teaching program. He directed the Physical Department of the Esisar from 1997 to 2006. From 2006 to 2009, he was the Director of Study of that same school. In 2010-2011, he was visiting professor at the École Polytechnique de Montréal in the Poly-Grames Microwave Research Center. Since 2014, he is Head of International Relations of Esisar. His first research topics concerned integrated optics on glass substrates for sensors applications. From 2004 to 2010, he actively participated to the creation of a French startup company specialized in optical sensors. In 2006, he changed his research activities to electromagnetic wave generation and propagation: antennas, array antennas, frequency selective surface and localized waves. He is the author and co-author of more than 80 journal papers, letters, and international conferences. He is co-author of 5 patents. His work on wall-paper blocking WiFi signals won several French awards.

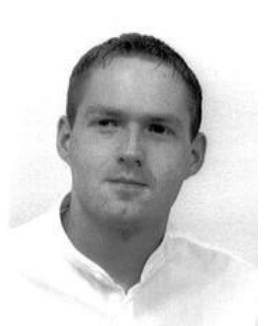

Etienne Perret received the Eng. Dipl. in electrical engineering from the Ecole Nationale Supérieure d'Electronique, d'Electrotechnique, d'Informatique, d'Hydraulique, et des Télécommunications, Toulouse, France, in 2002, and the M.Sc. and Ph.D. degrees from the Toulouse Institute of Technology, France, in 2002 and 2005, respectively, all in electrical engineering. From 2005 to 2006, he held a post-doc position at the Institute of Fundamental Electronics (IEF), Orsay, France. Since September 2006, Dr. Perret is Associate Professor in electrical engineering with the Grenoble Institute of Technology. In October 2014, he was elevated to Junior member of the "Institut Universitaire de France", an institution that distinguishes professors for their research excellence, as evidenced by their international recognition.

$\mathrm{He}$ is authored and co-authored of more than 120 technical conferences, letters and journal papers, and books. He is IEEE senior member and Technical Program Committee member of the IEEE International Conference on RFID. He was keynote speaker and the chairman of several international symposiums. His research activities cover the electromagnetic modeling of passive devices for millimeter and submillimeter-wave applications. His current research interests are in the field of wireless communications, especially radio frequency identification (RFID) and chipless RFID. His interests also involve advanced computer aided design techniques based on the development of an automated co-design synthesis computational approach. He was named one of MIT Technology Review's French Innovator's under 35 in 2013 for his work on chipless RFID. He is also the recipient of the French's Innovative techniques for the environment awards in 2013. 